Abstract E-118 Table 1 Institutional CAS outcomes versus CREST CAS outcomes

\begin{tabular}{lll}
\hline & $\begin{array}{l}\text { Institutional CAS } \\
\text { Outcomes (N=73) no. } \\
\text { of patients }(\% \pm \text { SE) }\end{array}$ & $\begin{array}{l}\text { CREST CAS Outcomes } \\
(\mathrm{N}=1262) \text { no. of } \\
\text { patients }(\% \pm \mathrm{SE})\end{array}$ \\
\hline Peri-procedural death & 0 & $9(0.7 \pm 0.2)$ \\
Peri-procedural stroke & 0 & $52(4.1 \pm 0.6)$ \\
Peri-procedural myocardial infarction & 0 & $14(1.1 \pm 0.3)$ \\
Any major ipsilateral stroke & $1(1.4 \pm 0.013)$ & $11(0.9 \pm 0.3)$ \\
$\begin{array}{l}\text { Primary end point (any peri- } \\
\text { procedural stroke, myocardial }\end{array}$ & $1(1.4 \pm 0.013)$ & $66(5.2 \pm 0.6)$ \\
infarction, or death or post- & & \\
procedural ipsilateral stroke) & & \\
\hline
\end{tabular}

Median follow up time was 17.4 months (IQR 6.8-29.5 months). No peri-procedural ( $<30$ days) stroke, MI, or death occurred in this cohort. Post-procedural stroke, including ipsilateral or contralateral anterior circulation and posterior circulation stroke, occurred in three patients (4.1\%). One experienced a major ipsilateral stroke at 10 months, in the setting of interrupted anticoagulation due to spontaneous subdural hematoma. Major posterior circulation stroke occurred in two patients, one at 5 weeks and one at five years postprocedure. Repeat ipsilateral intervention occurred in three patients (4.1\%), including one for acute in-stent thrombosis on the day of procedure in the setting of sub-therapeutic DAPT, despite being previously therapeutic. There were three $(4.1 \%)$ patient deaths in the first year post-procedure from metabolic encephalopathy, status epilepticus, and one unknown cause. End-points of data collected from our institution are compared to data reported after CAS in the CREST trial (table 1).

Conclusion Elective carotid artery stenting at our institution is a safe procedure, with rates of peri-procedural stroke, MI, and death lower than those reported in the CREST trial.

Disclosures A. Forrest: None. C. Sellers: None. P. Ramakrishnan: None. W. Leesch: None. F. Sanderson: None.

\section{E-119 SURPASS STREAMLINE FLOW DIVERTER USE IN TREATING CERVICAL CAROTID PSEUDOANEURYSMS: A CASE SERIES}

R Achey*, L Sheikhi, T Patterson, G Toth, N Zobenica Moore, M Bain. Neurological Institute, Cleveland Clinic, Cleveland, $\mathrm{OH}$

10.1136/neurintsurg-2020-SNIS. 151

Introduction Flow diverter stents have revolutionized endovascular treatment for cerebral aneurysms not amenable to coil embolization. Originally, the Pipeline embolization device (PED) emerged as a novel system for treating large/ giant, or wide-necked intracranial carotid artery (ICA) aneurysms from the petrous to superior hypophyseal segments, with later extension up to the terminus with small/ medium aneurysms. Recently, the Surpass streamline flowdiverter (SSFD) has shown promise for diversifying flowdiversion options for aneurysm treatment. The SSFD differs from PED by increasing pore density while maintaining high metal-surface-area to ensure uniform flow disruption between the parent artery and aneurysmal lumen. The SSFD also expands from $5 \mathrm{~mm}$ up to $7 \mathrm{~mm}$ further diversifying treatment possibilities. There are few reports of flowdiverter stents used for cervical carotid pseudoaneurysms in the literature. However, the SSFD appears uniquely well adapted to targeting these aneurysms given the system's increased diameter/length options and expandability. Here, we describe our group's experience with the SSFD as a stand-alone flow-diversion solution for cervical carotid pseudoaneurysms.

Materials and Methods Data pertaining to cervical pseudoaneurysms treated with SSFD were gathered retrospectively January 2019 to now. Data included age, aneurysm type (sidewall, fusiform), size, symptoms, number of stents placed, and SSFD dimensions. Indications for stent placement included enlarging aneurysm, worsening compressive symptoms, and TIA/stroke. Complications were noted at four time points post-operatively: $<24$ hours, 30 days, 6 months and one year. Aneurysm occlusion degree was characterized using SMART grading (0 - arterial, coherent inflow jet to 4 - complete aneurysm occlusion) immediately post-procedure and at one-year follow-up angiogram. Six month post-procedure MRI/MRA was used for interval aneurysm occlusion assessment.

Results Three patients underwent SSFD placement for cervical carotid pseudoaneurysms. Ages ranged from 55-78. Two patients had symptomatic sidewall aneurysms measuring $1.7 \times$ $2.1 \mathrm{~cm}$ and $1.8 \times 1.3 \mathrm{~cm}$. Both patients developed vocal hoarseness. One patient experienced vasovagal episodes while the other experienced TIAs. Another patient had an incidentally discovered fusiform aneurysm measuring $1.3 \times 1.5 \mathrm{~cm}$. There were no complications at any time point. One to three stents were deployed intra-operatively. Post-procedural SMART grade was 0 for two patients, and 2 for the third patient. One-year angiogram demonstrated SMART grade 3 occlusion with a minimally visualized neck remnant for one patient without parent artery stenosis. Six month MRI/MRA demonstrated total occlusion in another patient. Follow-up data was not available from the last patient as their initial intervention occurred $<6$ months ago. No stents migrated on follow-up imaging. The symptomatic patient had symptom resolution at one year.

Abstract E-119 Table 1 Characteristics of three patients treated with surpass for cervical carotid pseudoaneurysms

\begin{tabular}{|c|c|c|c|c|c|c|c|c|c|}
\hline Age & Sex & Aneurysm Size (mm) & Aneurysm Type & Symptoms & SSFD size $(\mathrm{mm})$ & No.Devices & Complications & Treatment Outcome & Symptom Resolution \\
\hline 77 & M & $17 \times 21$ & sidewall & hoarseness, vasovagal episodes & $\begin{array}{l}5 \times 50,5 \times 40 \\
5 \times 30\end{array}$ & 3 & none & SMART 3 at $1 \mathrm{yr}$ & resolved \\
\hline 78 & $\mathrm{~F}$ & $13 \times 15$ & fusiform & none & $5 \times 40$ & 1 & none & $\begin{array}{l}\text { no residual on } \\
\text { MRI/MRA at } 6 \text { mo }\end{array}$ & N/A \\
\hline 55 & M & $18 \times 13$ & sidewall & hoarseness, TIAs & $5 \times 50,5 \times 50$ & 2 & none & SMART 2 post-procedure & $\mathrm{N} / \mathrm{A}$ \\
\hline
\end{tabular}


Conclusions SSFD successfully treated three patients with cervical carotid pseudoaneurysms at our institution from 2019 to present with no complications, satisfactory angiographic appearance, and symptom resolution. Though further research is required to confirm these results, we demonstrate that SSFD appears both safe and effective for endovascular treatment of cervical carotid pseudoaneurysms.

Disclosures R. Achey: None. L. Sheikhi: None. T. Patterson: None. G. Toth: None. N. Zobenica Moore: None. M. Bain: None.

\section{E-120 THE UTILITY OF REPEAT COMPUTED TOMOGRAPHY ANGIOGRAM AFTER BLUNT CEREBROVASCULAR INJURY}

Z Aljuboori ${ }^{\star}, \mathrm{K}$ Meyer, R James, D Ding. Neurosurgery, University of Louisville, louisville, KY

\subsection{6/neurintsurg-2020-SNIS. 152}

Introduction Blunt cerebrovascular injury (BCVI) can lead to thromboembolic events. The necessity of short-term repeat vascular imaging after the initial diagnosis is controversial. The aim of this retrospective cohort study is to assess the utility of short-interval computed tomography angiography (CTA) after an initial diagnosis of BCVI.

Methods We retrospectively reviewed consecutive patients with BCVI managed at the University of Louisville from 2016-2019 who underwent short-term (1-3 weeks) repeat CTA after initial diagnosis. The exclusion criteria were age $<18$ years, penetrating injury, and previous neck irradiation. We collected baseline data and performed logistic regression analysis to identify predictors of BCVI imaging outcomes.

Results The study cohort comprised 38 patients (68\% male) with a mean age of 45 years. Motor vehicle accident (79\%) was the most common mechanism of injury, and $89 \%$ had cervical spine fractures. Unilateral VA and grade I dissection were the most common findings on initial CTA were a unilateral VA dissection (66\%) that was Biffl grade I (36\%). Antiplatelet therapy or anticoagulation was administered to $82 \%$ of patients after the initial diagnosis (table 1). Shift analysis showed a significant improvement in Biffl grades on repeat CTA $(p=0.0001)$ (figure 1$)$. Biffl grades I injuries were more likely to improve (relative risk ratio $[R R R]=3.6$, CI $95 \%$ $(1.02-13.1), \mathrm{p}=0.04)$, whereas grade IV injuries were more

\begin{tabular}{lll} 
Abstract E-120 Table 1 & Patients demographics & \\
\hline Variable & Percentage \\
\hline Gender & Males & $68 \%$ \\
Mechanism of injury & Fall & $21 \%$ \\
MVA & $79 \%$ & \\
Cervical spine fractures & $89 \%$ \\
First rib fractures & $16 \%$ \\
Early Stroke & $5 \%$ \\
Delayed Stroke & $0 \%$ \\
Vascular intervention & $8 \%$ \\
Received ASA or AC between 1st and 2nd CTA & $82 \%$ \\
Received ASA or AC after 2nd CTA & $82 \%$ \\
Abbreviations: AC; anticoagulation, ASA; aspirin, CTA; computed & \\
tomography angiogram, MVA; motor vehicle accident, FFH; fall from & \\
height & \\
\hline
\end{tabular}

likely to be stable $(\mathrm{RRR}=33$, CI 95\% (2.9-374), $\mathrm{p}=0.005)$ Ten BCVIs (26\%) completely resolved on repeat imaging (table 2). The rates of early ( $<2$ weeks) and delayed ( 2 weeks to 3 months) ischemia were $5 \%$ and $0 \%$, respectively. Endovascular stenting was performed in $8 \%$.

Conclusion Short-term repeat non-invasive vascular imaging can help to identify the evolution of BCVI. Repeat vascular imaging at short intervals can help to identify patients who may require endovascular intervention, but additional studies are necessary to clarify its role in the management of BCVI. Disclosures Z. Aljuboori: None. K. Meyer: None. R. James: None. D. Ding: None.

\section{E-121 SALVAGE ENDOVASCULAR STRATEGIES IN A UNIQUE DUPLICATED INTERNAL CAROTID ARTERY LEADING TO CATASTROPHIC DISSECTION AND FUSIFORM ANEURYSM}

Y Lodi*, B Pulgarin, Z Weiss, S Chin, V Reddy. Neurology, Neurosurgery and Radiology, Upstate Medical University/UHS-Wilson Medical Center, Johnson City, NY

10.1136/neurintsurg-2020-SNIS.153

Background Anatomical variance of duplicated internal carotid artery (ICA) is novel. However, duplication associated 